ESTUDO PILOTO

PILOT EXPERIMENT

\title{
Impacto da gestão do consumo de água no Hospital Estadual Américo Brasiliense: um estudo piloto
}

\author{
Impact of water consumption management at the \\ Brazilian American state hospital: a pilot study \\ Wilson Antônio Neri Júnior ${ }^{1}$, Fabiana Rossi Varallo², Tales Rubens De Nadai ${ }^{3}$
}

DOI: $10.21115 / J B E S . v 12 . n 1 . p 92-7$

\section{Palavras-chave:}

hospital, avaliação de programas e projetos de saúde, planejamento hídrico

\section{Keywords:}

hospital, program evaluation, water resources

\section{RESUMO}

Objetivos: Avaliar a redução da extração de água no poço artesiano após a instalação de medidores de vazão em pontos de distribuição hídrica num hospital. Métodos: Conduziu-se estudo piloto de intervenção do tipo antes e depois no Hospital Estadual Américo Brasiliense, localizado no interior do estado de São Paulo. A gestão da água iniciou-se em 2010 e realizou-se por meio do monitoramento de sete hidrômetros pré-equipados para sistema de telemetria, com relojoaria mecânica e sistema de turbinas, dos quais quatro foram instalados em 2016. Em março de 2017, foram instalados chuveiros e torneiras para limpeza com redutores de vazão em todo o hospital e arejadores em todas as torneiras para higienização das mãos. Analisou-se o impacto dos acessórios comparando-se a captação de água $\left(\mathrm{m}^{3}\right)$ do poço artesiano. Estimou-se o retorno financeiro após a implantação das medidas. Resultados: Observou-se redução entre 21\% e 42\% (600 e 1.444 m³) do total do consumo hídrico e em $8.000 \mathrm{~m}^{3}$ de água por ano na extração do aquífero. Com a economia entre 800 e $1.000 \mathrm{~m}^{3} /$ mês de água, obteve-se retorno dos investimentos em 13 dias da implantação dos acessórios, pois o montante investido para compra e instalação dos equipamentos foi de 10.955 mil reais e a economia advinda da redução do consumo hídrico foi de 24.302 mil reais. Conclusão: A instalação de medidores de vazão é custo-efetiva para economia de água e financeira em hospitais. As melhorias contribuem para aumentar a credibilidade do desenvolvimento de projetos sustentáveis e a viabilidade de investimentos em ações ambientais.

\section{ABSTRACT}

Objectives: Assess the reduction of water extraction in the artesian well after installation of flow restrictors in points of hydric distribution in a hospital. Methods: A pilot pre-post interventional study was carried out in Américo Brasiliense Hospital State, localized in the province of São Paulo (Brazil). Water management began in 2010. The monitoring was performed with seven water flow meters with telemetry system, with watchmaking and turbines of which four were installed in 2016. March 2017, flow restrictors on cleaning sinks and showers were installed, as well as aerators for hand basins. The impact of accessories was assessed comparing the water capitation $\left(\mathrm{m}^{3}\right)$ in artesian well before and after interventions. Financial payback was estimated after implementation of the accessories. Results: Total hydric consumption decrease by $21 \%$ a $42 \%$ (600 a 1,444 $\mathrm{m}^{3}$ ) and reduced the extraction of aquifer in $8,000 \mathrm{~m}^{3}$ of water/year. With the aid of approximately $1,000 \mathrm{~m}^{3} /$ month of water economy, the hospital had payback thirteen days after the implementation of the accessories, since the valor invested buy and install of the equipment was $R \$ 10.955,00$ reais and the save regarding the decrease of hydric consume was $\mathrm{R} \$ 24.302,00$ reais. Conclusion: Flow restrictors installation in critical points of hydric distribution is cost-effective to reduce water consumption and costs in hospitals. Improvements performed contribute to raise the credibility of sustainability projects and the viability of investment in environmental actions.

\footnotetext{
Recebido em: 20/02/2020. A provado para publicação em: 07/04/2020.

1. Hospital Estadual Américo Brasiliense, São Paulo, SP, Brasil.

2. Departamento de Ciências Farmacêuticas, Faculdade de Ciências Farmacêuticas de Ribeirão Preto, Universidade de São Paulo, Ribeirão Preto, SP, Brasil.

3. Departamento de Saúde Coletiva, Faculdade de Medicina de Bauru, Universidade de São Paulo, Bauru, SP, Brasil.

Nome da instituição onde o trabalho foi executado: Hospital Estadual Américo Brasiliense.

Informações sobre auxílios recebidos sob a forma de financiamento, equipamentos ou medicamentos: $\bigcirc$ trabalho foi conduzido sem quaisquer tipos de auxílios.

Congressos onde o estudo foi apresentado: Este estudo foi apresentado no I Seminário Regional Hospitais Saudáveis Hospital Estadual Américo Brasiliense.

Autor correspondente: Tales Rubens de Nadai. Alameda Dr. Octávio Pinheiro Brisolla, 9-75, Jardim Brasil, Bauru, SP, Brasil. CEP: 17012-901. Telefone: +55 (14) 3226-6512. E-mail: trnadai@usp.br
} 


\section{Introdução}

Os hospitais são grandes consumidores de água, representando até 3,5\% da despesa anual em instituições certificadas que participam do Sistema de Avaliação dos Hospitais de Ensino (Gomes et al., 2016).

Os fatores que desempenham grande impacto no consumo de água em instituições hospitalares compreendem: número de leitos e unidades de enfermarias, tempo da construção, acesso à água e serviços gerais dentro da estrutura e gerenciamento de políticas institucionais (Verlicchi et al., 2010; WHO, 2010).

Segundo a Declaração Universal dos Direitos da Água (ONU, 1992), a gestão da água impõe um equilíbrio entre os imperativos de sua proteção e as necessidades de ordem econômica, sanitária e social. Por se tratar de um recurso natural limitado e dotado de valor econômico (Brasil, 1997), todos os indivíduos e países devem se engajar num esforço coletivo para desenvolver estratégias que visem evitar o desperdício e contribuir para o uso consciente desse recurso.

O uso racional da água em instituições tornou-se um diferencial competitivo para as empresas modernas e, muitas vezes, a disponibilidade hídrica é o fator determinante na decisão de investimentos em novas instalações e ampliações (Ribeiro et al., 2015).

O gerenciamento desse recurso requer o exercício da gestão da demanda de forma a complementar a gestão da oferta (Gonçalves, 2006). Para tanto, são requeridos mudança de comportamento dos usuários, emprego de tecnologias que promovam a redução do consumo e o reuso (Gonçalves, 2006). Desse modo, aumenta-se a eficiência da utilização, bem como da qualidade na produção de bens e serviços.

Tendo em vista que as instituições de saúde buscam atingir padrões de qualidade em seus processos, o desenvolvimento de práticas de ecoeficiência, como requisito de qualidade, é um diferencial para uma empresa ou instituição pública para a mitigação de impactos ambientais (Toledo \& Demajorovic, 2006). No entanto, poucas são as iniciativas voltadas à implementação de ações para o gerenciamento e utilização racional da água em instituições de saúde (Mcgain \& Naylor, 2014).

Nesse contexto, o presente estudo teve como objetivos avaliar a redução da extração de água no poço artesiano e estimar o retorno financeiro após a instalação de medidores de vazão em pontos de distribuição hídrica num hospital público e geral do interior do estado de São Paulo.

\section{Métodos}

\section{Desenho epidemiológico e local do estudo}

Realizou-se um estudo do tipo experimental, aberto, não randomizado, com grupo único de comparação antes e depois, no Hospital Estadual Américo Brasiliense (HEAB), durante o período de janeiro de 2010 a agosto 2018, para avaliar o impacto da gestão hídrica no consumo e extração de água na instituição.
O HEAB é uma instituição pública, de média complexidade, com capacidade para 94 leitos, localizada no interior do estado de São Paulo. É um hospital referência que atende 23 municípios do Departamento Regional de Saúde III (DRS III), nas seguintes especialidades: geriatria, neurologia, clínica médica, infectologia e unidade de terapia intensiva adulta. Com relação às especialidades cirúrgicas, são atendidos casos de nefrologia/urologia, oftalmologia, ortopedia/traumatologia, gastroenterologia, cirurgia geral e torácica, otorrinolaringologia, plástica e ginecologia.

\section{Coleta de dados: intervenção e monitoramento}

O controle do consumo de água no complexo hospitalar foi iniciado em janeiro de 2010. Diariamente, o volume de água registrado em três hidrômetros pré-equipados para sistema de telemetria, com relojoaria mecânica e sistema de turbinas, era anotado pela equipe de manutenção e registrado em planiIha de controle. Os hidrômetros mensuravam o consumo da água utilizada nas atividades de assistência à saúde e outras atividades de apoio, tais como: produção de alimentos, higiene e limpeza, desinfecção de equipamentos médicos e uso de sanitários.

Em 2015, devido à estratégia institucional relacionada ao projeto de eficiência hídrica, verificou-se a necessidade da adoção de novos sistemas para acompanhamento do consumo em todos os pontos de distribuição, permitindo à instituição aperfeiçoar a gestão, direcionar a implementação de intervenções técnicas para reduzir o consumo e avaliar os resultados obtidos após os investimentos.

Mediante estudos da distribuição hidráulica e das instalações existentes, identificaram-se pontos que necessitariam da instalação de novos medidores de consumo, possibilitando controles mais efetivos da relação entre captação e consumo na instituição. De acordo com o levantamento realizado em meados de agosto de 2016, quatro novos medidores de vazão foram instalados, totalizando sete pontos de monitoramento. A partir de então, no projeto piloto, foi possível mensurar o consumo de água das enfermarias de moléstias infecciosas, clínica cirúrgica e outros setores de apoio. Optou-se por esses setores, uma vez que são representativos no consumo geral e, após a instalação dos novos hidrômetros, foi possível comparar o consumo depois das intervenções realizadas.

Com o intuito de reduzir o consumo de água $\left(\mathrm{m}^{3}\right)$ e a extração de água do poço artesiano $\left(\mathrm{m}^{3}\right)$, estabeleceram-se os pontos críticos de distribuição hídrica no hospital para implantação de tecnologias, a saber: aproximadamente chuveiros (115 unidades), torneiras de higienização das mãos (580 unidades) e todas as torneiras utilizadas para limpeza (53 unidades). Optou-se por esses pontos, uma vez que o maior consumo de água está atribuído à higiene (banho e limpeza) (Victorian Government Department of Health, 2018). 
As tecnologias empregadas foram: 1) para os chuveiros e torneiras, foram utilizados redutores de vazão resistentes à temperatura entre 0 e $100^{\circ} \mathrm{C}$; 2) para as torneiras de higienização das mãos com retorno automático, foram instalados arejadores nos pontos de consumo, o qual permite a distribuição da água com jato otimizado e disperso.

Em março de 2017, foram iniciadas as instalações de todos os acessórios previstos no planejamento. Diariamente, as medidas foram realizadas nos sete hidrômetros e anotadas em planilhas. As anotações obtidas após a instalação dos acessórios (finalizadas em agosto de 2018) foram comparadas com as anotações realizadas antes da implantação das tecnologias supracitadas. Os dados foram apresentados em estatística descritiva.

\section{Resultados}

No período de 2013 a 2016, a média do consumo anual de água no HEAB foi de $38.723 \mathrm{~m}^{3}$ (Tabela 1). Após a instalação dos acessórios, observou-se redução de $21 \%\left(7.972 \mathrm{~m}^{3}\right)$ do total do consumo hídrico e de $21 \%$ a $42 \%$ pontualmente quando verificado mês a mês. Em alguns meses, a redução atingiu $42 \%\left(1.444 \mathrm{~m}^{3}\right)$, totalizando $8.000 \mathrm{~m}^{3}$ de água não extraída do aquífero de Serra Geral, quando comparado com o ano-base (2016).

A partir do mês seguinte à instalação dos acessórios (mar./2017), verificou-se que a captação de água no poço artesiano ficou abaixo da meta estabelecida, exceto no mês de dez./2017, quando houve a implantação de grandes jardins, e em set./2018, por avaria no sistema hidráulico para a correção de vazamentos (Tabela 2).

Os redutores de vazão nos chuveiros e torneiras permitiram a redução na vazão de água de $18 \mathrm{~L} /$ min para $5 \mathrm{~L} /$ min e de $50 \mathrm{~L} / \mathrm{min}$ para $15 \mathrm{~L} / \mathrm{min}$, respectivamente, sem interferir na rotina diária dos atendimentos. Já para as torneiras de higienização das mãos com retorno automático, observou-se redução do consumo de 1,2 L/min para 0,25 L/min. Portanto, houve redução do consumo em aproximadamente $80 \%$ nas torneiras destinadas a higiene das mãos. Com a economia média entre 800 e $1.000 \mathrm{~m}^{3} / \mathrm{mês}$ de água, obteve-se retorno dos investimentos estimado em 13 dias após a implantação dos acessórios, pois o montante investido para a compra dos acessórios e instalação foi de $R \$ 10.955,00$ reais e a redução do consumo hídrico foi de R\$24.302,00.

Antes da instalação dos equipamentos, o consumo de água nas enfermarias de clínica médica e moléstias infecciosas apresentava-se acima das médias estabelecidas na maioria dos anos analisados (Figura 1). Após a instalação dos redutores, verificou-se queda importante no consumo de ambos os setores, obtendo-se valores abaixo das médias estabelecidas para cada um.

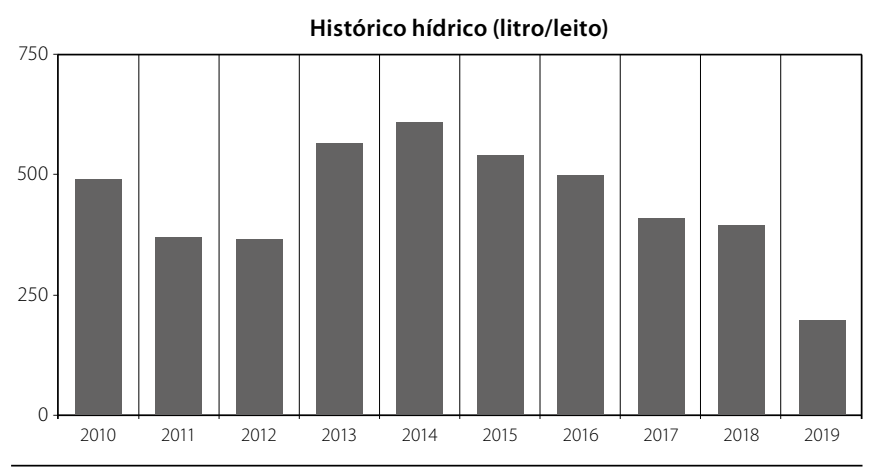

Figura 1. Comparação do consumo de água $\left(\mathrm{m}^{3}\right)$ no complexo hospitalar após a instalação de redutores de fluxo hídrico nos chuveiros e torneiras para limpeza e higienização das mãos, relacionado aos leitos disponíveis.

Tabela 1. Histórico do consumo $\left(\mathrm{m}^{3}\right)$ de água e porcentagem de economia hídrica, por mês, após a instalação dos acessórios no Hospital Estadual Américo Brasiliense.

\begin{tabular}{|c|c|c|c|c|c|c|c|c|c|c|c|c|c|c|c|c|}
\hline ANO & Jan. & Fev. & Mar. & Abr. & Maio & Jun. & Jul. & Ago. & Set. & Out. & Nov. & Dez. & Total & $\mathrm{m}^{3} / \mathrm{m}^{2}$ & $\mathrm{~m}^{3} / \mathrm{L}$ & $\begin{array}{l}\text { L/leito- } \\
\text { dia }\end{array}$ \\
\hline 2010 & 3.156 & 2.517 & 3.266 & 3.070 & 3.266 & 3.847 & 3.815 & 3.846 & 3.653 & 2.797 & 2.719 & 3.293 & 39.245 & 1,35 & 178 & 496 \\
\hline 2012 & 3.044 & 2.455 & 679 & 2.875 & 3.844 & 3.328 & 3.891 & 1.668 & 3.416 & 3.043 & 1.943 & 2.660 & 32.846 & 1,13 & 134 & 371 \\
\hline 2013 & 3.364 & 2.753 & 2.835 & 4.396 & 3.732 & 3.191 & 1.153 & 3.843 & 2.962 & 3.605 & 3.280 & 3.239 & 38.353 & 1,32 & 206 & 573 \\
\hline 2016 & 3.042 & 2.753 & 3.459 & 3.516 & 3.503 & 3.387 & 3.523 & 3.146 & 2.967 & 3.289 & 2.998 & 2.908 & 38.491 & 1,33 & 182 & 504 \\
\hline 2017 & 3.055 & 2.668 & 2.878 & 2.538 & 2.588 & 2.370 & 2.740 & 2.179 & 2.368 & 2.721 & 2.442 & 3.075 & 31.622 & 1,09 & 149 & 414 \\
\hline Red & $0,4 \%$ & $-3 \%$ & $-17 \%$ & $-28 \%$ & $-26 \%$ & $-30 \%$ & $-22 \%$ & $-31 \%$ & $-20 \%$ & $-17 \%$ & $-19 \%$ & $6 \%$ & $-18 \%$ & & & \\
\hline 2018 & 1.935 & 2.128 & 2.342 & 2.611 & 2.686 & 2.475 & 3.011 & 2.815 & 3.065 & 2.640 & 2.476 & 2.335 & 30.519 & 1,05 & 144 & 400 \\
\hline
\end{tabular}

RED: redução alcançada. 
O maior consumo de água na enfermaria de moléstias infecciosas ocorreu em função do perfil epidemiológico atendido. O período de hospitalização é maior nesse setor quando comparado ao da clínica cirúrgica, a qual tem caráter de hospital-dia. Desse modo, espera-se que maior volume de água seja utilizado para rotinas de higiene ao paciente.

\section{Discussão}

Nossos dados corroboram que economia de 21\% a 42\% (600 a $1.444 \mathrm{~m}^{3}$ ) no consumo de água pode ser obtida por meio da implantação de medidas simples, que não exigem inovações ou pesquisas (Mcgain \& Naylor, 2014). Entretanto, a inserção de equipamentos hidráulicos e acessórios ainda é escassa nas torneiras em um hospital de ensino universitário (Buono, 2018).

Além da instalação de medidores para controle e registro de dados, aplicação de restritores em lavatórios e chuveiros, outras medidas, tais como auditorias, verificação de vazamentos e substituição de água por outros desinfetantes, também podem ser implantados para redução do consumo de água (Jehle et al., 2008).

A busca ativa diária de vazamentos e equipamentos danificados, com intervenção imediata para correção dos problemas, levantamento das condições da rede interna e externa, reservatórios e equipamentos, compreende rotinas que devem ser empregadas no planejamento da gestão hídrica em hospitais. Ilha et al. (2006) observaram que o conserto de vazamentos detectados que ainda estavam em execução reduziu, consideravelmente, o consumo de água e, consequentemente, os gastos com esse insumo. Portanto, acompanhamento em tempo real do consumo de água na instituição faz-se necessário, na expectativa de que possam ser feitas intervenções imediatas em caso de perdas (Ribeiro et al., 2015).
A instalação de hidrômetros em cada setor de uma instituição hospitalar também é uma medida que melhora o controle do consumo de água, pois a setorização do uso tem como objetivo a obtenção de dados mais precisos (Silva, 2011), além de permitir o conhecimento dos patamares do volume utilizado, possibilitando intervenção corretiva tão logo problemas que geraram alterações sejam detectados (Sautchúk, 2004). Tal medida já está prevista em novos empreendimentos e visa minimizar as dificuldades atuais para contabilizar custos hospitalares pelo desconhecimento do consumo de água nas diferentes grandes áreas hospitalares (Sautchúk, 2004; Bittar, 2015).

Não obstante, o desenvolvimento de ações educativas também pode ser inserido no projeto de manejo hídrico hospitalar. Há consenso sobre a necessidade de treinar os recursos humanos hospitalares, com o intuito de conscientizar e contribuir para a aquisição de conhecimento sobre conservação, reuso e reciclagem da água (D’Alessandro et al., 2016). Embora os profissionais da saúde reportem a importância da redução do consumo, na prática são escassas as atitudes efetivas destinadas para melhorar o manejo hídrico (D'Alessandro et al., 2016).

Desse modo, os estabelecimentos e profissionais da saúde não devem subestimar suas influências potenciais em ações de sustentabilidade (Buffoli et al., 2015). Assim, o indicador de mensuração de intensidade do uso da água é uma medida da eficiência da utilização dos recursos, com o intuito de maximizar a produtividade, minimizando a intensidade do consumo (Siplon, 2013).

Ressalta-se que esse indicador consta em programas de certificação ambiental para os estabelecimentos de saúde. Por exemplo, no Canadá, estabelece-se como melhor prática o consumo máximo de $3,11 \mathrm{~m}^{3} / \mathrm{m}^{2} / a n o$. No Reino Unido, documento técnico estabelece o consumo entre 0,90 e 1,38 m³ $/ \mathrm{m}^{2}$.

Tabela 2. Volume real e a meta $\left(\mathrm{m}^{3}\right)$ da extração de água do poço artesiano após a instalação dos acessórios

\begin{tabular}{|c|c|c|c|c|c|c|c|c|c|c|c|}
\hline Mês & $\mathrm{C}^{\circ}$ & \multicolumn{7}{|c|}{ Volume real $\left(\mathrm{m}^{3}\right)$} & $\begin{array}{l}\text { Meta } \\
\text { Meta }\end{array}$ & \multicolumn{2}{|c|}{ Volume real $\left(\mathrm{m}^{3}\right)$} \\
\hline Jan. & 27 & 3.156 & 3.255 & 3.044 & 3.364 & 3.658 & 3.027 & 3.042 & 2.747 & 3.055 & 1.935 \\
\hline Mar. & 27 & 3.266 & 2.333 & 679 & 2.835 & 3.598 & 3.573 & 3.459 & 2.347 & 2.878 & 2.342 \\
\hline Abr. & 27 & 3.070 & 3.535 & 2.875 & 4.396 & 3.280 & 1.962 & 3.516 & 2.760 & 2.538 & 2.611 \\
\hline Jun. & 25 & 3.847 & 3.290 & 3.328 & 3.191 & 3.843 & 3.335 & 3.387 & 2.986 & 2.370 & 2.475 \\
\hline Jul. & 26 & 3.815 & 1.491 & 3.891 & 1.153 & 3.652 & 3.497 & 3.523 & 2.529 & 2.740 & 3.011 \\
\hline Ago. & 29 & 3.846 & 845 & 1.668 & 3.843 & 3.825 & 3.587 & 3.146 & 2.492 & 2.179 & 2.815 \\
\hline Set. & 31 & 3.653 & 3.653 & 3.416 & 2.962 & 2.962 & 3.461 & 2.967 & 2.823 & 2.368 & 3.065 \\
\hline Total & & 41.255 & 35.379 & 34.858 & 40.366 & 43.157 & 36.903 & 38.491 & 31.508 & 31.622 & 30.519 \\
\hline
\end{tabular}


No Brasil, a Companhia de Saneamento Básico do Estado de São Paulo (Sabesp, 2012) preconiza que o consumo de água em hospitais deve obedecer à seguinte equação: $\left(2,9 \times n^{\circ}\right.$ de funcionários $)+\left(11,8 \times n^{\circ}\right.$ de bacias $)+\left(2,5 \times n^{\circ}\right.$ de leitos $)+280$. A faixa de vazão do uso típico de água em instalações hospitalares, considerando o número de leitos, é de 491 a 983 litros/ unidade/dia, sendo a vazão típica de 567 litros/unidade/dia (Tomaz, 2000).

A utilização de programas de gerenciamento da água no combate ao uso excessivo de água potável está em constante evolução em relação as técnicas sustentáveis, as quais vem sendo adotadas em várias edificações, com resultados positivos (Buono, 2018).

Gomes et al. (2016) observaram que os Hospitais de Ensino do Estado de São Paulo (HE-SP), em 2013, informaram ao Sistema de Avaliação dos Hospitais de Ensino (SAHE) a média de 326,06 a 422,27 m³/leito operacional/ano, com o valor mínimo de 138,79 m²/leito operacional/ano máximo de 917,26 m²/leito operacional/ano para hospitais de 77 a 1.200 leitos operacionais. Os autores concluem que na média os Hospitais de Ensino apresentam um bom desempenho no consumo de água, próximo de dados internacionais (Gomes et al., 2016).

A avaliação do consumo de água no Hemocentro Coordenador de Palmas (TO) demonstrou que houve oscilações no período de 2011 a 2012 (entre $44 \mathrm{~m}^{3}$ e $366 \mathrm{~m}^{3}$ ), as quais não estavam relacionadas ao número de funcionários, público externo em eventos, número de bolsas processadas e número de atendimentos aos candidatos doadores de sangue e medula óssea (Ribeiro et al., 2015). Os autores inferiram que as variações de consumo se deveram às perdas físicas associadas à baixa qualidade da rede hidráulica e dos aparelhos hidrossanitários (Ribeiro et al., 2015).

Os dados de consumo registrados nas enfermarias de moléstias infecciosas e clínica cirúrgica corroboram com a literatura, quando mostram que o tipo de atendimento, além do número de leitos e a área construída, influencia na utilização de água (García-Sanz-Calcedo et al., 2017). Paradoxalmente, hospitais de porte pequeno podem consumir mais água quando comparados com os de grande porte (García-Sanz-Calcedo et al., 2017).

Portanto, evitar o desperdício deixou de ser questão puramente econômica. A atividade hospitalar está entre as inúmeras modalidades de serviços que pode desempenhar um papel central na mitigação ou expansão dos impactos socioambientais (Toledo \& Demajorovic, 2006).

O uso racional da água em instituições, de modo geral, tornou-se um diferencial competitivo para as empresas modernas e, muitas vezes, a disponibilidade hídrica é o fator determinante na decisão de investimento em novas instalações e ampliações (Ribeiro et al., 2015).

A experiência exitosa da gestão hídrica no Hospital Estadual Américo Brasiliense é um incentivo para que outras instituições de saúde públicas se motivem a implantar medidas de baixo custo que minimizem o desperdício de água em suas regiões.

\section{Conclusão}

A instalação de medidores de vazão nos pontos críticos de distribuição hídrica é custo-efetiva para a economia de água e financeira em hospitais, pois contribui para a economia de 800 a $1.000 \mathrm{~m}^{3} / \mathrm{mês}$ de água, com retorno dos investimentos aproximado em 13 dias da implantação dos acessórios, variando com a sistemática de implantação e a tarifa local da concessionaria responsável pelo abastecimento. O montante investido para a compra e instalação dos acessórios foi de R\$10.955,00, e a economia obtida pela redução do consumo hídrico foi de R\$ 24.302,00 mensais. Desse modo, sugere-se que as melhorias realizadas contribuem para aumentar a credibilidade do desenvolvimento de projetos sustentáveis e a viabilidade de investimentos em ações ambientais.

\section{Referências bibliográficas}

Bittar OJNV. Saúde: medir para conhecer. In: Isosaki M, Gandolfo AS, Jorge AL, Evazian D, Castanheira FA, Bittar OJNV. Indicadores de Nutrição Hospitalar. São Paulo: Atheneu; 2015. p. 1-12.

Brasil. Lei no 9.433, de 08 de janeiro de 1997. Institui a Política Nacional de Recursos Hídricos, cria o Sistema Nacional de Gerenciamento de Recursos Hídricos, regulamenta o inciso XIX do art. 21 da Constituição Federal, e altera o art. $1^{\circ}$ da Lei n 8.001, de 13 de marco de 1990, que modificou a Lei no 7.990, de 28 de dezembro de 1989. Diário Oficial da União, Poder Executivo, Brasília, DF, 09 jan. 1997.

Buffoli M, Capolongo S, Di Noia, Gherardi G, Gola M. Healthcare sustainability evaluation systems. In: Capolongo S, Bottero MC, Buffoli M, Lettieri E, eds. Improving Sustainability During Hospital Design and Operation: A Multidisciplinary Evaluation Tool. Cham: Springer; 2015.

Buono LN. Diagnóstico de uso de água no Hospital Universitário de Londrina: estudo de caso [dissertação]. Londrina: Universidade Tecnológica Federal do Paraná (UTFPR); 2018.

D'Alessandro D, Tedesco P, Rebecchi A, Capolongo S. Water use and water saving in Italian hospitals. A preliminary investigation. Ann Ist Super Sanita. 2016;52(1):56-62.

García-Sanz-Calcedo J, López-Rodrigues F, Yusaf T, Al-Kassir W. Analysis of the Average Annual Consumption of Water in the Hospitals of Extremadura (Spain). Energies. 2017;10:479.

Gomes AM, Bittar OJNV, Fernandes AD. Sustainability in health: water and its consumption. Revista de Gestão em Sistemas de Saúde - RGSS. 2016;5(1):76-85.

Gonçalves RF. Uso Racional da Água em Edificações. 1a ed. Rio de Janeiro: ABES; 2006. 352p.

Ilha MSO, Nunes SS, Salermo LS. Programa de conservação de água em hospitais: estudo de caso do Hospital das Clínicas da Universidade Estadual de Campinas. Ambiente Construído. 2006;6(1):91-7.

Jehle K, Jarrett N, Matthews S. Clean and green: saving water in the operating theatre. Ann R Coll Surg Engl. 2008;90(1):22-4.

Mcgain F, Naylor C. Environmental sustainability in hospitals: a systematic review and research agenda. J Health Serv Res Policy. 2014;19(4):245-52.

Organização das Nações Unidas (ONU). Declaração de Dublin sobre água e desenvolvimento sustentável. Nova York, EUA: ONU; 1992. 
Ribeiro MS, Sampaio SMR, Kiperstok A, Cardoso LF. Avaliação do consumo de água no Hemocentro Coordenador de Palmas (TO). In: Brasil. Ministério da Saúde. Secretaria de Atenção à Saúde. Departamento de Atenção Hospitalar e de Urgência. Gestão ambiental: ecoeficiência e produção mais limpa nas práticas da hemorrede pública nacional: Curso de Especialização em Gestão Ambiental com Ênfase em Produção Mais Limpa para a Hemorrede Pública Nacional: artigos de conclusão do curso. 1a ed. Brasília: Ministério da Saúde; 2015.

SABESP. Norma Técnica 181: Dimensionamento do ramal predial de água, cavalete e hidrômetro - Primeira ligação. Rev. 4; 2017.

Sautchúk CA. Formulação de diretrizes para implantação de programas de conservação de água em edificações [dissertação]. São Paulo: Escola Politécnica, Universidade de São Paulo; 2004.

Silva PCA. Reserva hídrica: Aquífero Guarani e seu uso sustentável. Monografia (especialização) - Curso em Legislativo e Políticas Públicas, Câmara dos Deputados, Centro de Formação, Treinamento e Aperfeiçoamento (Cefor); 2011.

Siplon J. California Hospital Association, "We are in this together" 2013. Available from: http://www.calhospital.org/sites/main/files/ fileattachments/jim_siplon_final_app.pdf. Accessed on: 15 jul. 2015.
Toledo AF, Demajorovic J. Atividade hospitalar: impactos ambientais e estratégias de ecoeficiência. Revista de Gestão Integrada em Saúde do Trabalho e Meio Ambiente. 2006;1(2):1-23.

Tomaz P. Previsão de consumo de água: Interface nas Instalações Prediais de Água e Esgoto com os Serviços Públicos. São Paulo: Navegar; 2000.

Verlicchi P, Galletti A, Petrovic M, Barceló D. Hospitals effluents as a source of emerging pollutants: an overview of micropollutants and sustainable treatment options. J Hydrol. 2010;389:416-28.

Victorian Government Department of Health. Guidelines for water reuse and recycling in Victorian health care facilities. Non-drinking application. Available from: http://docs.health.vic.gov.au/docs/ doc/8F90C51C3C55F3D4CA257AA600049D29/\$FILE/water_reuse_and_ recycling.pdf. Accessed on: Nov 9, 2019

WHO - World Health Organization. Water safety in buildings. Geneva: WHO; 2010. 